\title{
Transdermal delivery of FITC-Dextrans with different molecular weights using radiofrequency microporation
}

\author{
Guk Young Ahn', Hae-Seok Eo², Dongwon Kim² and Sung-Wook Choi ${ }^{1 *}$ (D)
}

\begin{abstract}
Background: Transdermal delivery is of great importance for the effective delivery of bioactive or therapeutic agents into a body. The microporation device based on radiofrequency can be used to enhance delivery efficiency by removing the epidermis layer.
\end{abstract}

Methods: The micropores were developed on pig skin and human cadaver skin with dermal and epidermal layers by the microporation device. The regeneration of micropores in the human cadaver skin caused by microporation was confirmed using an optical microscope and haematoxylin/eosin (H\&E) staining. The permeability of fluorescein isothiocyanate-dextrans (FITC-dextrans) with different molecular weights through the pig and human cadaver skins were measured using Franz diffusion cell.

Results: The optical image and histological analysis confirmed that the micropores on the skin were recovered over time. The enhanced permeability through micropores was confirmed by Franz diffusion cell. The lower molecular weight of FITC-dextran permeated more on both human and pig skin. In addition, the permeation rate was higher in pig skin than in human skin.

Conclusions: We believe that the microporation device can be used as a potential technique for effective transdermal drug delivery.

Keywords: Microporation device, Human cadaver skin, Transdermal drug delivery

\section{Background}

Transdermal drug delivery is an attractive alternative that addresses the limitations of oral and parenteral routes of drug administration; it enables controlled release and long-term systemic drug delivery through the skin, avoids hepatic first-pass effect, avoids gastrointestinal drug degradation, reduces discomfort and trauma resulting from hypodermic injections, and prevents safety hazards associated with dangerous medical waste from needles $[1,2]$. However, the currently

\footnotetext{
* Correspondence: choisw@catholic.ac.kr

'Department of Biomedical-Chemical Engineering, The Catholic University of Korea, 43 Jibong-ro Wonmi-gu, Bucheon-si, Gyeonggi-do 14662, Republic of Korea

Full list of author information is available at the end of the article
}

available transdermal systems are limited by low drug permeability across the skin due to the lipophilic barrier function of the stratum corneum (SC), the outermost skin layer of the skin [3-5]; the methods favor low molecular mass, and lipophilic molecules, making it difficult to exploit the transdermal route for large and water-soluble molecules such as proteins and peptides. To overcome these limitations, microporation devices that act as therapeutic vehicles for delivering drug molecules across the skin barriers have been developed. This method utilizes promising strategies that have made a significant impact in transdermal delivery [6], they include: iontophoresis, microneedles, sonophoresis, radiofrequency (RF), and 
chemical enhancers [7-11]. Radiofrequency is a wellknown medical technique with expanded application in electrosurgery for laparoscopic medical procedures and in dermatology, for sebaceous glands ablation in acne treatment $[12,13]$, and treatment of photoaging skin symptoms, including wrinkles and hyperpigmentation [14]. To improve drug permeability, micropore ablation using RF is performed by passing an alternating electrical current through the skin at a frequency higher than $100 \mathrm{kHz}$. Micropores are produced by the arrangement of microelectrodes on the skin at precise dimensions, then RF energy generated by alternating currents induces ionic vibrations between electrodes with positive or negative charges. These vibrations heat the skin tissue, triggering water vapor and cell ablation, forming microchannels from the SC through to the outer dermis. Immediately after formation, the microchannels are filled with interstitial fluid, making them hydrophilic, and a suitable drug delivery system [15]. The effectiveness of the RF microporation technique in transdermal drug delivery of macromolecules and hydrophilic agents such as peptides, hormones, and vaccines has been demonstrated extensively in previous studies [16-18]. However, in vitro investigations are very few. In this study, we investigated the effectiveness of RF microporation technology in vitro, by evaluating the permeation of fluorescent-conjugated dextran (FITC-dextran) of various molecular weights through the human and pig skin after microporation, using a transdermal diffusion cell system. In addition, we evaluated the regeneration of SC after microporation. Because of the recent animal protection rules which have restricted animal experiments [19], we used human cadaver skin as an alternative to animals. Human cadaver skin has been confirmed as alive skin tissue through analysis of its morphology and enzymes, and its usefulness as an alternative skin membrane in drug permeation experiments has been verified [20].

\section{Materials and methods \\ Materials}

FITC-dextran molecular weights; 4, 10, and $20 \mathrm{kDa}$ molecular weight were purchased from Sigma (St. Louis, MO, USA). Dulbecco's Phosphate-Buffered Saline (DPBS) was purchased from Welgene (Gyeongsan, Korea). The skin culture medium was purchased from Biopredic International (Saint-Grégoire, France). 10\% neutral buffered formalin was purchased from Hisko (Gunpo, Korea).

\section{Equipment}

The skin poration was performed using the microporation device (LG electronics, Korea). The measurements of the transepidermal water loss (TEWL) were taken using the Tewameter (CourageKhazaka Electronic $\mathrm{GmbH}$, Cologne, Germany) to evaluate the skin barrier function after microporation. The Transdermal diffusion cell system (DHC-6TD, LOGAN instrument, USA) was used to assess FITCdextran permeability in vitro.

\section{In vitro human cadaver skin regeneration after microporation}

Human cadaver skins (fresh human full-thickness skin disc, $\varnothing 12 \sim 20 \mathrm{~mm}$ ) were supplied by Biopredic International (Saint-Grégoire, France). The skins were from the abdomen of a 39-year-old caucasian woman (BMI: 24). One day before the experiment, the skin was stabilized in the skin culture medium. Barrier integrity was examined using Tewameter to confirm the poration of human cadaver skin using a microporation device. TEWL measurements were performed before and $30 \mathrm{~min}$ after microporation. Microporation was performed 2, 5, and 10 times on each sample. Each microporated sample was incubated in the skin culture medium. Pore closure examination was performed using an optical microscope (BX43, Olympus, Japan) immediately (time 0 ), 4,8 , and $24 \mathrm{~h}$ following device application. Each skin pores was captured using a microscope and the obtained images were analyzed using Image ${ }^{\circ}$ software (National Institutes of Health, Bethesda, USA).

\section{Histological characterization of skin pore closure}

After the pore closure examination, each sample was stored in a $10 \%$ neutral buffered formalin awaiting haematoxylin/eosin (H\&E) staining. These studies were carried out at the Hisko (Gunpo, Korea). H\&E stained images were analyzed using Image $J^{\circ}$ software.

\section{In vitro transdermal delivery after microporation}

The human and pig skin permeability of FITC-dextran (M.W. 4, 10, and $20 \mathrm{~K}$ ) was measured using the Transdermal diffusion cell system. FITC-dextran solution was prepared at $1 \mathrm{mM}$ in PBS. Human skins (Full-thickness human skin) were supplied by HansBiomed. Corp. (Seoul, Korea). The human skins were from the back or thigh of a 24-year-old woman. Pig skins (Micropig ${ }^{\circ}$ Franz cell membrane) were supplied by Medi Kinetics Co., Ltd. (Pyeongtaek, Korea). Microporation was performed 2 times on each sample. TEWL measurements were taken $30 \mathrm{~min}$ after microporation of the human and pig skin. The human and pig skin samples were placed in the receiver chamber of the Transdermal diffusion cell system with the SC facing up and the donor chamber was fixed in place. 


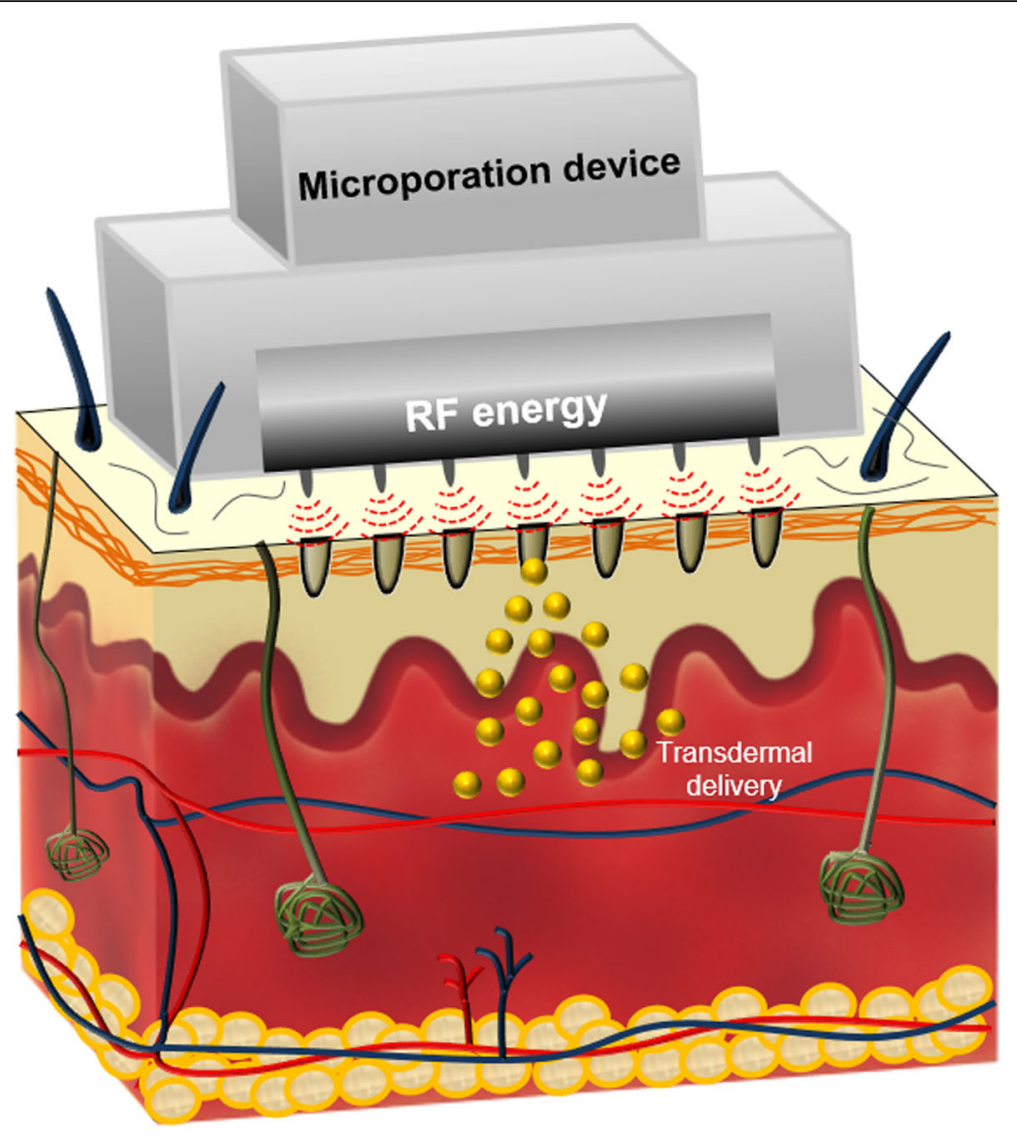

Fig. 1 Schematic illustration of transdermal delivery after the application of the microporation device

The receptor chamber was filled with PBS. FITC-dextran solution $(600 \mu \mathrm{L})$ was added to the donor chamber. The Transdermal diffusion cell system was protected from light. The receptor phase was collected immediately (time 0 ) and 2, 4, 8, 12, and $24 \mathrm{~h}$. This receptor phase solution was transferred to a 96-well plate and the skin-permeated FITC-dextran was measured using a microplate reader (Molecular Devices, Co. Ltd., Sunnyvale, CA, USA) at 405 $\mathrm{nm}$.

\section{Statistics}

Quantitative data are presented as the mean \pm standard deviation, and comparisons were carried out using one-way ANOVA (Systat Software Inc., Chicago, IL, USA). Differences were considered statistically significant at $p<0.05$.

\section{Results}

Figure 1 shows a schematic illustration of transdermal delivery after the application of the microporation device. The skin was treated with the RF-based

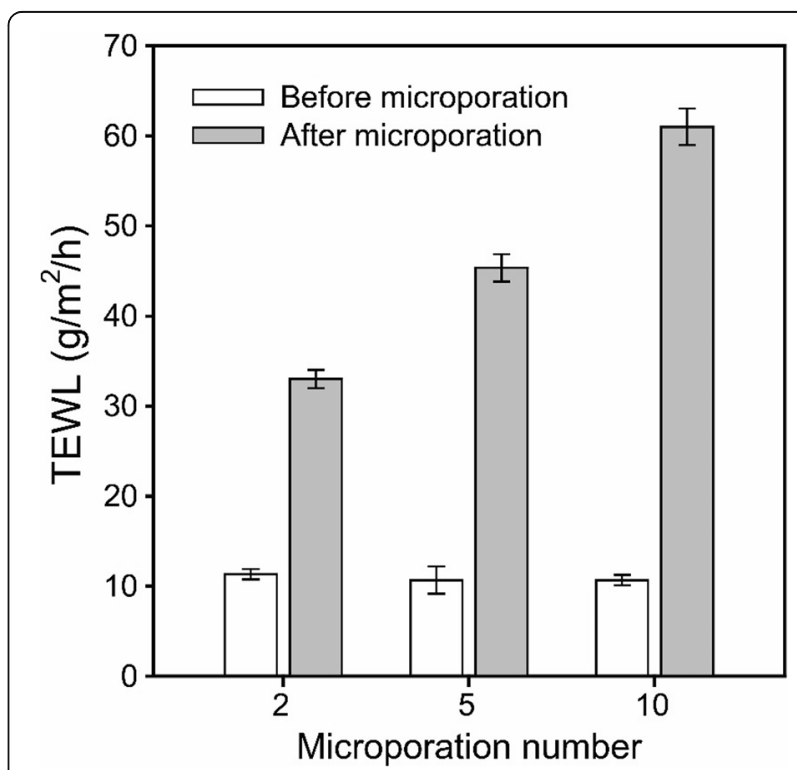

Fig. 2 TEWL values before and immediately after microporation on human cadaver skin according to the number of times microproration was done $(n=3)$ 
microporation device, inducing micropore ablation through alternating electrical current. This device can generate microchannels from the SC through to the outer dermis and allow transdermal delivery of active agents.

Figure 2 shows TEWL values measured before and immediately following the treatment of human cadaver skin with the microporation device. To confirm the extent of skin damage and regeneration after microporation, porations were performed 2, 5, and 10 times. TEWL measurements were taken to assess the integrity of the skin barrier and the extent of SC disruption. After microporation, the TEWL values of the human cadaver skin increased from $11.3 \pm 0.6$ to $33.0 \pm 1.0,10.7 \pm 1.5$ to $45.3 \pm 1.5$, and $10.7 \pm 0.6$ to $61.0 \pm 2.0 \mathrm{~g} / \mathrm{m}^{2} / \mathrm{h}$ when the microporation was performed 2, 5, and 10 times, respectively.

Figure 3 shows a magnified image of human cadaver skin with microchannels formed by the microporation device $(250 \mathrm{kHz})$ and the skin closure over time. Immediately after 2,5 , and 10 times microporations, $\quad 97.14 \pm 32.39, \quad 80.57 \pm 15.51, \quad$ and $82.39 \pm$ $16.49 \mu \mathrm{m}$ sizes of micropores were formed on the skin, respectively. $4 \mathrm{~h}$ after microporation, the sizes of the micropore decreased slightly. After $8 \mathrm{~h}$, the micropore became more distorted from the previous circle, suggesting that the micropores were closing. After 24 $\mathrm{h}$ the micropores were either closed or reduced further to smaller sizes, $37.50 \pm 20.41,50.57 \pm 18.07$, and $58.58 \pm 14.99 \mu \mathrm{m}$, for 2,5 , and 10 times microporation, respectively. These results indicate that healing
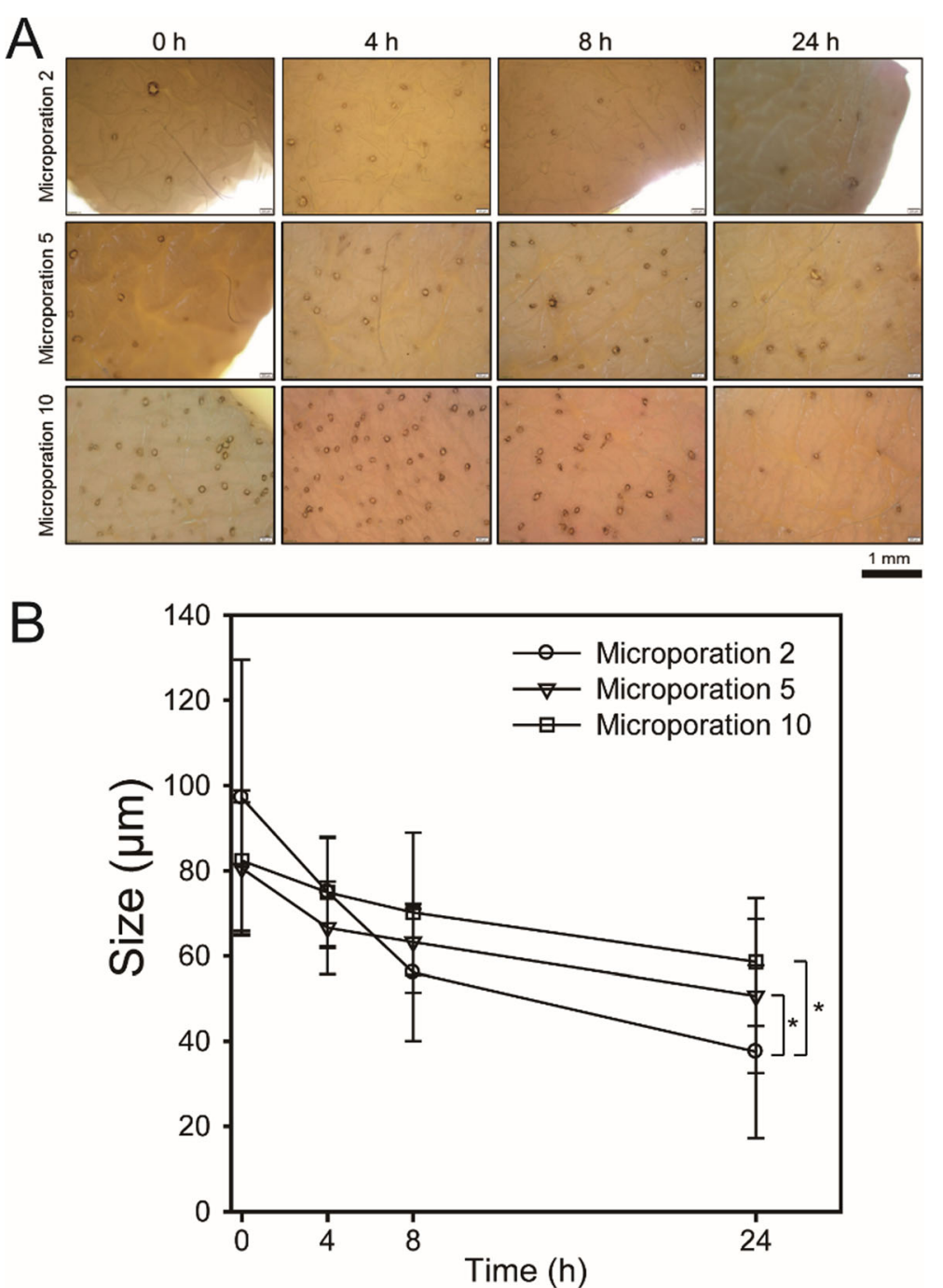

Fig. 3 a Microscopy images of micropore and $\mathbf{b}$ quantified data using Image after 0 (immediately), 4, 8, and $24 \mathrm{~h}$ according to the number of times microproration was done $\left(n=150,{ }^{*}: p<0.05\right)$ 


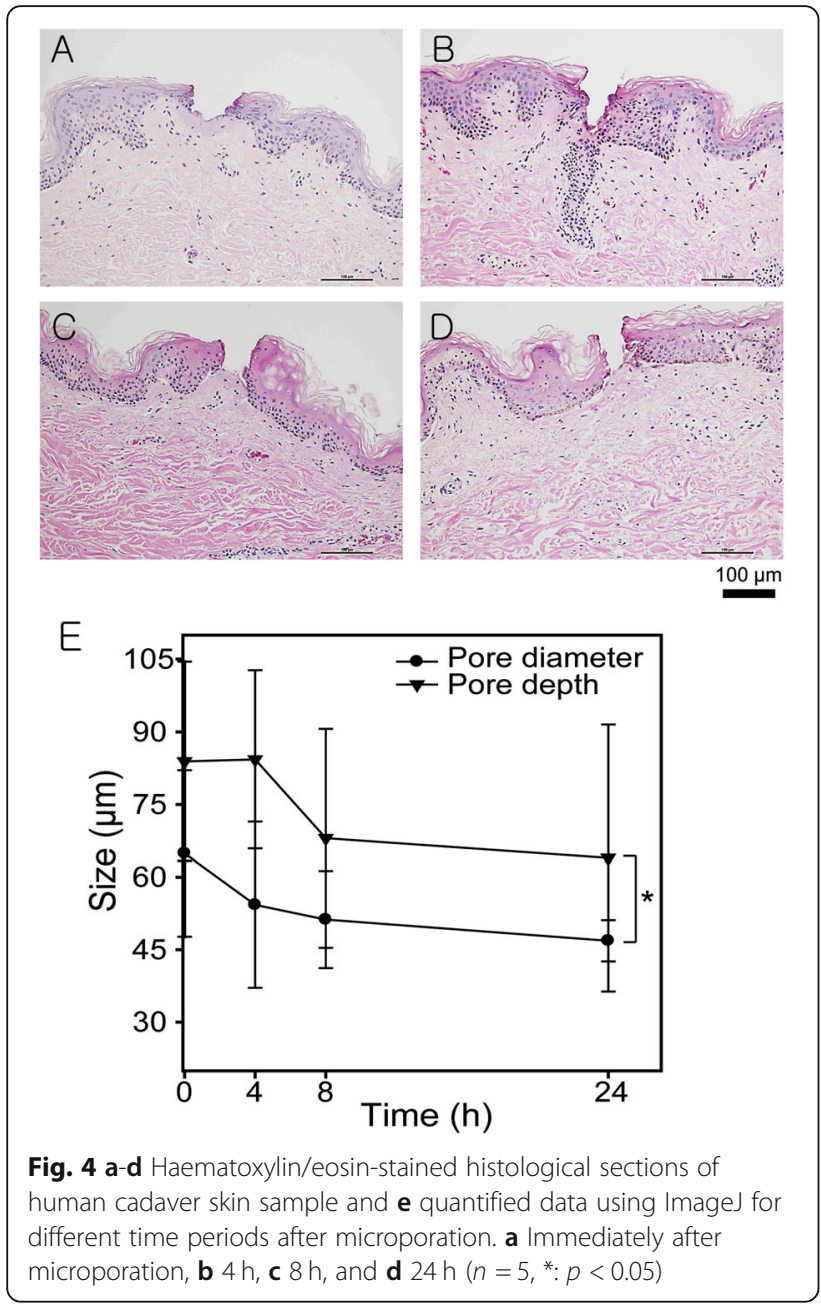

and regeneration occurred in the human cadaver skin after the microporation.

Figure 4 shows the histological analysis of micropores in human cadaver skin. The micropore formed at the SC through to the epidermis, enabling drug delivery. $24 \mathrm{~h}$ after microporation, the pore sizes reduced from $64.88 \pm 17.20 \mu \mathrm{m}(0 \mathrm{~h})$ to $48.85 \pm 4.27 \mu \mathrm{m}(24 \mathrm{~h})$, and the pore depths reduced from $83.91 \pm 20.56 \mu \mathrm{m}(0 \mathrm{~h})$ to $63.95 \pm 27.61 \mu \mathrm{m}$ (24 h). These results suggest that fibroblasts proliferate and migrate under the SC, and regenerate the skin.

Table 1 shows TEWL values measured immediately after the microporation of human and pig skins. Increased TEWL values are evidence of micropores formation. These results are in agreement with a previous study which suggested that the microporation using radiofrequency causes disruption of the SC, enabling the percutaneous permeation of the FITCdextran [21]. Figure 5 shows the cumulative amount of the FITC-dextran (M.W. $4 \mathrm{~K}, 10 \mathrm{~K}$, and $20 \mathrm{~K}$ ) permeated through the human, and pig skin after microporation. After $24 \mathrm{~h}$, the cumulative permeation of FITC-dextran M.W. $4 \mathrm{~K}$
Table 1 TEWL values before and immediately after microporation of the human and pig skin

\begin{tabular}{llcc}
\hline & & $\begin{array}{c}\text { TEWL }\left(\mathrm{g} / \mathrm{m}^{2} / \mathrm{h}\right) \text { of } \\
\text { before microporation }\end{array}$ & $\begin{array}{c}\text { TEWL }\left(\mathrm{g} / \mathrm{m}^{2} / \mathrm{h}\right) \text { of } \\
\text { after microporation }\end{array}$ \\
\hline FD 4 K & Human 1 & 12 & 35 \\
& Human 2 & 15 & 27 \\
& Human 3 & 14 & 32 \\
& Pig 1 & 11 & 23 \\
& Pig 2 & 11 & 23 \\
& Pig 3 & 12 & 21 \\
\hline FD 10 K & Human 1 & 19 & 42 \\
& Human 2 & 15 & 41 \\
& Human 3 & 15 & 40 \\
& Pig 1 & 14 & 33 \\
& Pig 2 & 14 & 37 \\
& Pig 3 & 14 & 38 \\
\hline FD 20 K & Human 1 & 19 & 42 \\
& Human 2 & 17 & 37 \\
Human 3 & 16 & 39 \\
Pig 1 & 15 & 44 \\
Pig 2 & 14 & 44 \\
& Pig 3 & 15 & 36 \\
\hline
\end{tabular}

was $10.61 \pm 1.68$ and $13.44 \pm 0.18 \mu \mathrm{M}$ in human and pig skin, respectively. The cumulative permeation of FITC-dextran $10 \mathrm{~K}$ was $4.82 \pm 0.32 \mu \mathrm{M}$, in the human skin and $5.92 \pm$ $0.46 \mu \mathrm{M}$ in the pig skin. With FITC-dextran $20 \mathrm{~K}$, cumulative permeation in the human skin was $2.53 \pm 0.48 \mu \mathrm{M}$, and $3.34 \pm 0.19 \mu \mathrm{M}$ in the pig skin, which significantly lower than FITC-dextran $4 \mathrm{~K}$.

\section{Discussion}

In this work, the human cadaver skin was treated to confirm the skin regeneration after the application of the microporation device. Microporations were performed 2, 5, and 10 times and the skin damage was confirmed using the TEWL measurement. Normally, the skin loses very little water loss whereas damaged skin loses more [22]. The increased TEWL values which were proportional to the number of microspores indicate SC disruption and formation of microchannels in the skin.

The micropores of the human cadaver skin were regenerated according to the time. Keratinocytes adjacent to the wound site induced by the microporation in the epidermis undergo a series of modifications that allow their proliferation and migration to the wound. These modifications include degradation of hemidesmosomes attached to the dermis, degradation 

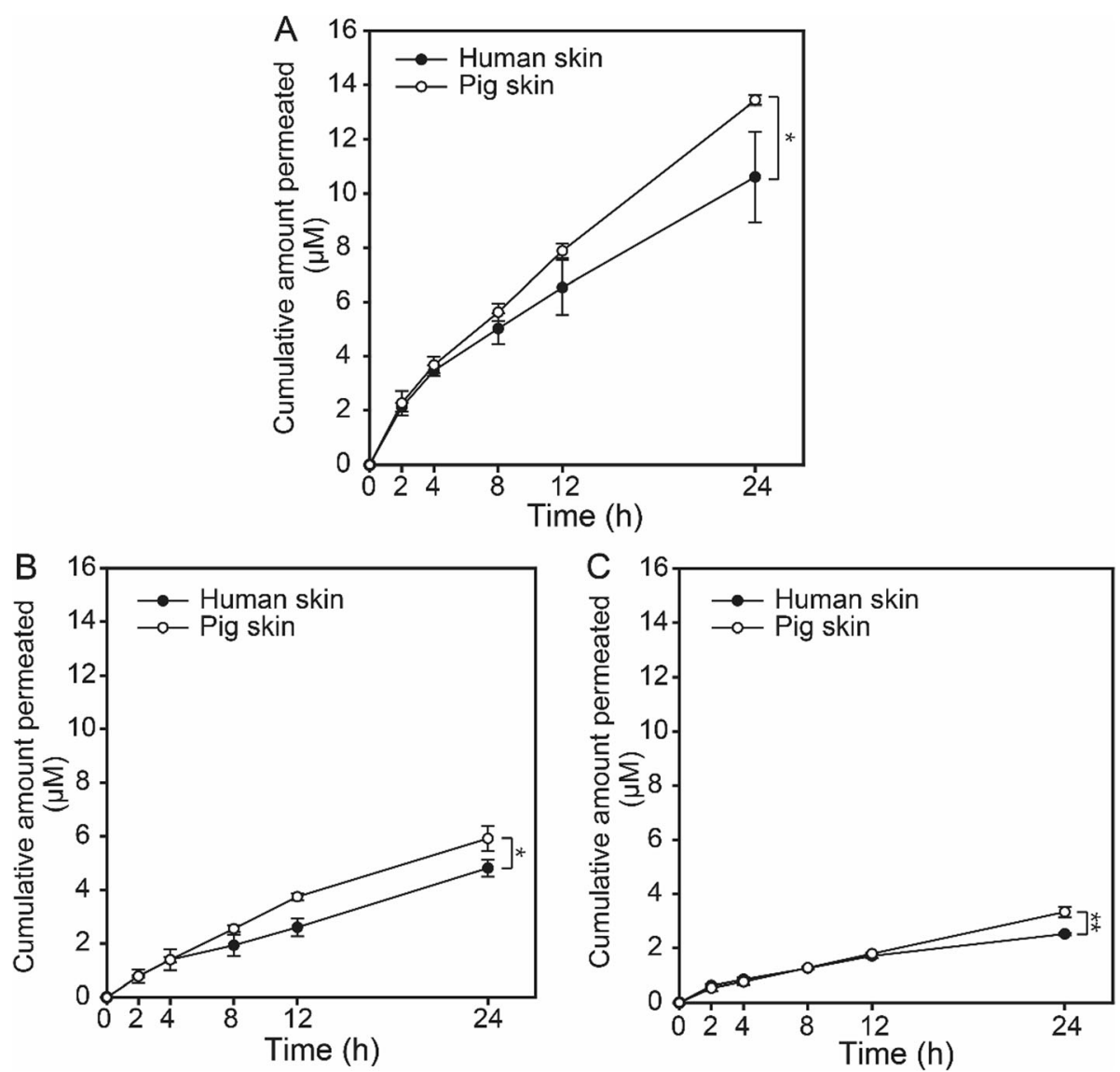

Fig. 5 Cumulative amount of FITC-dextran permeated after microporation on the human and pig skin for FITC-dextran M.W.. a M.W. 4 K, b M.W. $10 \mathrm{~K}$, and c M.W. $20 \mathrm{~K}$. * shows a significant difference between the M.W. $4 \mathrm{~K}$ and M.W. $20 \mathrm{~K}$ groups $\left(n=3\right.$, $\left.^{*}: p<0.05,{ }^{* *}: p<0.01\right)$

of desmosomes linked to adjacent cells, contraction of intracellular tonofilament, and lamellipodia formation. The regeneration process of the skin is regulated by growth factors such as keratinocyte growth factor and fibroblast growth factor, which trigger the proliferation and migration of cells [23, 24]. However, the results did not show significant regeneration of micropore compared to previous in vivo studies which reported significant regeneration [25]. These results could be attributed to the differences in the in vivo and in vitro skin nutrients supply; with in vivo studies, the skin tissue is supplied nutrients directly from the blood vessels, but the human cadaver skin is supplied from the media, therefore, the skin tissue regeneration efficiency is low [26-28]. However, considering the trauma and unethical practices associated with animal experiments, in vitro studies with human cadaver skin offers an ethical and viable alternative. Although we didn't conduct assay for inflammation, we could not find any inflammation symptoms such as redness, blisters, cracked, and thickening.
The dextran has the advantage in terms of low cytotoxicity, excellent biocompatibility, and watersolubility. Therefore, it is suitable as model molecules with different molecular weights for transdermal drug delivery [29]. In our present study, microporated skin permeated insufficient amount of $20 \mathrm{~K}$ FITC-dextran, suggesting that enhancement of permeability was unsuccessful with macromolecules of more than $20 \mathrm{KM}$.W.. In addition, microporated pig skin permeated larger amounts of FITC-dextran than human skin, this can be attributed to the differences in structure between the human and pig skin; the pig skin layer is thinner (SC, $8-13 \mu \mathrm{m})$ than the human skin $(\mathrm{SC}, 10-17 \mu \mathrm{m})$ and the pig hair follicle is longer $(38-71 \mu \mathrm{m})$ than the human hair follicle $(18 \mu \mathrm{m})$. These structural differences can significantly affect FITC-dextran delivery due to even after microporation [30]. Based on these results a difference of approximately $10-15 \%$ between the amounts of drugs permeated through the human skin and pig skin models should be considered during human clinical trials. 


\section{Conclusions}

In summary, we demonstrated effective enhancement of skin permeability and delivery of macromolecules lower than $20 \mathrm{KM}$.W., and regeneration of human cadaver skin after microporation with an RF-based microporation device. In vitro studies with human cadaver skin is a viable, ethical, and economical alternative for in vivo animal studies. In the future, we plan to investigate the possibility of enhancing the delivery of other macromolecular drugs such as peptides and siRNAs using the RFbased microporation device. We will also conduct further studies on the regeneration of human cadaver skin and evaluate the possibilities of clinical trials.

\section{Acknowledgements}

Not applicable.

\section{Authors' contributions}

SWC designed and coordinated the research. GYA primarily conducted research. HSE and DK helped to porate the skin and to measure TEWL. All authors read and approved the final manuscript.

\section{Funding}

This study was supported by the LG Electronics.

\section{Availability of data and materials}

For data requests, please contact the authors.

\section{Ethics approval and consent to participate} Not applicable.

\section{Consent for publication}

All authors have consented to the submission of this manuscript for publication.

\section{Competing interests}

The authors declare that they have no competing interests.

\section{Author details}

'Department of Biomedical-Chemical Engineering, The Catholic University of Korea, 43 Jibong-ro Wonmi-gu, Bucheon-si, Gyeonggi-do 14662, Republic of Korea. ${ }^{2}$ LG electronics, 19 Yangjae-daero 11-gil, Seocho-gu, Seoul 06772, Republic of Korea.

\section{Received: 14 October 2020 Accepted: 25 November 2020}

Published online: 09 December 2020

\section{References}

1. Kumar SV, Tarun P, Kumar TA. Transdermal drug delivery system for nonsteroidal anti inflammatory drugs: a review. Indo AM J Pharm. 2013;3(5): 3588-605.

2. Naik A, Kalia YN, Guy RH. Transdermal drug delivery: overcoming the skin's barrier function. Pharmaceut Sci Tech. 2000;3(9):318-26.

3. Prausnitz MR, Langer R. Transdermal drug delivery. Nat Biotechnol. 2008; 26(11):1261-8

4. Nair SS. Strategies to improve the potential of transdermal devices by enhancing the skin permeation of therapeutic entities. J. Drug deliv. Ther. 2019;9(3-S):972-6.

5. Herwadkar A, Banga AK. Peptide and protein transdermal drug delivery. Drug Discov Today Technol. 2012;9(2):147-54

6. Singh TRR, Garland MJ, Cassidy CM, Migalska K, Demir YK, Ryan SAE, Woolfson D, Donnelly RF. Microporation techniques for enhanced delivery of therapeutic agents. Recent Pat Drug Deliv Formul. 2010;4(1):1-17.
7. Vemulapalli V, Bai Y, Kalluri H, Herwadkar A, Kim H, Davis SP, Friden PM, Banga AK. In vivo iontophoretic delivery of salmon calcitonin across microporated skin. J Pharm Sci. 2012;101(8):2861-9.

8. Sachdeva V, Banga AK. Microneedles and their applications. Recent Pat. Drug Deliv. Formul. 2011;5(2):95-132.

9. Whiteside PJD, Chininis JA, Schellenberg MW, Qian C, Hunt HK. Increased epidermal laser fluence through simultaneous ultrasonic microporation. Proc of SPIE. 2016;9706:97061E-6.

10. Ibrahim O, Munavalli GS, Dover JS. Radiofrequency with microneedling. Advances in Cosmetic Surgery. 2018;1:109-15.

11. Pathan $I B$, Setty $C M$. Chemical penetration enhancers for transdermal drug delivery systems. Trop J Pharm Res. 2009;8(2):173-9.

12. Curley SA. Radiofrequency ablation of malignant liver tumors. Ann Surg Oncol. 2003;10:338-47.

13. Gold MH, Biron JA. Treatment of acne scars by fractional bipolar radiofrequency energy. J Cosmet Laser Ther. 2012;87(6):172-8.

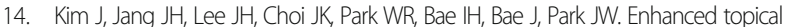
delivery of small hydrophilic or lipophilic active agents and epidermal growth factor by fractional radiofrequency microporation. Pharm Res. 2012;29(7):2017-29.

15. Lee WR, Shen SC, Sun CK, Aljuffali IA, Suen SY, Lin YK, Wang JJ, Fang JY. Fractional thermolysis by bipolar radiofrequency facilitates cutaneous delivery of peptide and siRNA with minor loss of barrier function. Pharm Res. 2015;32(5):1704-13.

16. Levin G, Gershonowitz A, Sacks H, Stern M, Sherman A, Rudaev S, Zivin I, Phillip M. Transdermal delivery of human growth hormone through RFmicrochannels. Pharm Res. 2005:22(4):550-5.

17. Mitragotri S. Mechanical disruption of skin barrier for vaccine delivery. Drug Deliv Syst. 2012;27(3):202-12.

18. Sintov AC, Krymberk I, Daniel D, Hannan T, Sohn Z, Levin G. Radiofrequencydriven skin microchanneling as a new way for electrically assisted transdermal delivery of hydrophilic drugs. J Control Release. 2003;89(2):311-20.

19. Doke SK, Dhawale SC. Alternatives to animal testing: a review. Saudi Pharm J. 2015;23(3):223-9.

20. Kano S, Todo H, Furui K, Kenichi S, Tokudome Y, Hashimoto F, Kojima H, Sugibayashi K. Comparison of several reconstructed cultured human skin models by microscopic observation: their usefulness as an alternative membrane for skin in drug permeation experiments. AATEX. 2011:16(2):51-8.

21. Rissmann R, Oudshoorn MHM, Hennink WE, Ponec M, Bouwstra JA. Skin barrier disruption by acetone: observations in a hairless mouse skin model. Arch Dermatol Res. 2009;301(8):609-13.

22. Banga AK. Microporation applications for enhancing drug delivery. Expert Opin Drug Deliv. 2009;6(4):343-54.

23. Hrabchak C, Flynn L, Woodhouse KA. Biological skin substitutes for wound cover and closure. Expert Rev Med Devices. 2006;3(3):373-85.

24. Santoro MM, Gaudino G. Cellular and molecular facets of keratinocyte reepithelization during. Exp Cell Res. 2005:304(1):274-86.

25. Kam Y, Sacks H, Kaplan KM, Stern M, Levin G. Radio frequencymicrochannels for transdermal delivery: characterization of skin recovery and delivery window. Pharmacol Pharm. 2012;3(1):20-8.

26. Qi P, Caoa M, Songa L, Chena C, Liua M, Li N, Wua D, Penga J, Hub G, Zhao J. The biological activity of cationic liposomes in drug delivery and toxicity test in animal models. Environ Toxicol Pharmacol. 2016;47:159-64.

27. Middelkoop E, Bogaerdt AJ, Lamme EN, Hoekstra MJ, Brandsma K, Ulrich MMW. Porcine wound models for skin substitution and burn treatment. Biomaterials. 2004;25(9):1559-67.

28. Salunkhe SS, Bhatia NM, Pokharkar VB, Thorat JD, Bhatia MS. Topical delivery of idebenone using nanostructured lipid carriers: evaluations of sunprotection and anti-oxidant effects. Int J Pharm Investig. 2013;43(4):287-303.

29. Huang S, Huang G. Preparation and drug delivery of dextran-drug complex. Drug Deliv. 2019;26(1):252-61.

30. Boudry I, Blanck O, Cruz C, Blanck M, Vallet V, Bazire A, Capt A, Josse D, Lallement G. Percutaneous penetration and absorption of parathion using human and pig skin models in vitro and human skin grafted onto nude mouse skin model in vivo. J Appl Toxicol. 2008;28(5):645-57.

\section{Publisher's Note}

Springer Nature remains neutral with regard to jurisdictional claims in published maps and institutional affiliations. 